\title{
Me miró mal. Agresividad y violencia entre niñas
}

\section{Looked at me bad. Agressiveness and violence among girls}

\author{
Carolina del Fresno**
}

\section{Resumen}

El presente artículo se propone visibilizar las particulares prácticas de niñez (Fernández, 2014) de un grupo de niñas indagando sobre una cierta forma de violencia y agresividad que las caracteriza y su vínculo con las condiciones socio-territoriales del barrio en el que conviven, desde una perspectiva de género. Este ensayo es el resultado de una experiencia de trabajo con un grupo de niñas de 10 a 14 años en el marco de un dispositivo sociocomunitario perteneciente al estado municipal. Luego de una elaboración en torno a los conceptos de territorio, agresividad y género como categoría transversal, como herramientas para analizar la práctica, se introducen las nociones de ternura y sororidad como posibles mecanismos restitutivos.

Palabras claves: Niña - Género - Violencia - Territorio - Agresividad

\section{Abstract}

This article aims to make render visible the particular practices of childhood (Fernández, 2014) in a group of girls by digging in a certain form of violence and aggressiveness that characterizes them; also, investigate their link with socio-territorial conditions in the neighbourhood where they live, from a gender perspective. This essay is the result of a work-experience with a group of girls aged from 10 to 14 in the context of a socio-community system in a Municipality. After an elaboration around the concepts of territory, aggressiveness and gender as cross-sectional categories, tools to analyze the practice, the notions of tenderness and sorority were introduced as possible restorative mechanisms.

Keywords: Girl - Gender - Violence Territory - Agressiveness

\footnotetext{
* El presente artículo fue escrito sobre la base del Trabajo Final Integrador de la Carrera de Especialización en Psicología Clínica Institucional y Comunitaria "Violencia y Agresividad. La difícil tarea de ser niña hoy" del que soy autora y fuera dirigido por la Ps. Sandra Gerlero.

** Especialista en Psicología Clínica Institucional y Comunitaria.
} 


\section{Introducción}

Entre los años 2013 y 2018, me desempeñé como educadora social en un barrio de la zona sur de la ciudad. En el proceso de trabajo, junto a otras compañeras y compañeros nos fuimos encontrando con que los espacios propuestos para niños y niñas de entre 10 y 14 años eran tomados por los varones que expulsaban violentamente a las niñas. Entendimos que esto se daba porque los niños se saben desde muy pequeños, por su condición de varones, dueños del espacio público. Por ello, definimos construir un dispositivo que sea exclusivo para niñas y lo pensamos como dispositivo porque tendía a hacer visible las prácticas de niñez (Fernández, 2014) que este grupo marginado lograba desplegar.

En el texto "La invención de la niña", Ana María Fernández (1994) afirma que la niña es una construcción socio-histórica que difiere en tanto mujer del niño, y que no todas las niñas de una sociedad se inscriben en similares prácticas de niñez, ni tampoco responden a un universo de significaciones común. Asimismo, las niñas han sido invisibles en la historia, en los estudios sociales, en el lenguaje, etc. El universo semántico y social de niñez omite particularidades de la niña en tanto suele tomar como modelo al niño. Dicho silencio es tributario de la invisibilidad de los estereotipos y de las vulnerabilidades a las que las niñas están expuestas y son esos mismos estereotipos los que pueden considerarse organizadores de la vida como niñas, primero, y posteriormente como mujeres adultas, al modo de una discriminación encubierta (Giberti, 1992).

Es por esto que se hace necesaria la ge- neración de espacios de visibilización en los ámbitos comunitarios y en el diseño de políticas de Estado, pero también dentro de la academia.

En el encuentro con las niñas, en el espacio de taller diseñado, el elemento que más nos llamó la atención fue el particular modo de vincularse entre ellas y con otras niñas que no asistían al espacio, teñido por grandes niveles de agresividad y violencia, direccionados al menosprecio de los caracteres vinculados al aspecto físico y la sexualidad, es decir al cuerpo y al placer. Es por esto que nos propusimos indagar y comprender estos modos de relacionarse y su vínculo con las condiciones socio-territoriales del barrio en el que conviven, desde una perspectiva de género.

En función de la práctica profesional e implicación con el ámbito de realización del trabajo aspiramos a llevar a cabo un estudio descriptivo comprensivo de carácter cualitativo y flexible. Para esto acudimos al uso de la observación no participante, que fue directa y no controlada. Además, en las reuniones grupales, elaboramos cuadernos de campo en los cuales se registraron los dichos textuales de las niñas en torno a sus pares, su cuerpo, la relación con los varones, lo femenino, el ser mujer. Sobre estos ejes también se realizaron una serie de entrevistas semiestructuradas a las niñas.

Se trabajó con el análisis de los contenidos de los discursos y observaciones elaborados bajo una modalidad de construcción de conocimiento emergente y espiralada o dialéctica. La concepción de la construcción social del género (Lagarde, 2004; Butler 2007) nos permitió llevar a cabo un trabajo de deconstrucción de las categorías 
tradicionales de género, que sostienen los enunciados. Es importante aclarar que los nombres y otros datos identitarios a los que se hace referencia a lo largo del trabajo, han sido modificados arbitrariamente para el resguardo de la confidencialidad.

A partir de ese análisis, hemos podido construir algunas coordenadas para pensar este fenómeno a la luz de los conceptos de territorio, violencia, crueldad y agresividad, siempre atravesados por la perspectiva de género. En ese marco, entendemos que la subjetividad femenina es un proceso multideterminado y que el género nunca aparece en su forma pura, sino entrecruzado con otros aspectos determinantes de la vida de las personas (Burin, 1996), la posibilidad de pensar esta violencia en clave de apropiación del discurso patriarcal, pero tomando los modos de la territorialidad actual, nos permitió delinear alguna senda para comenzar a reflexionar sobre esta problemática.

Fue importante también, en el marco de tanta violencia, introducir dos elementos que funcionan a contrapelo de esas lógicas: por el lado del sujeto la ternura como fundamento de la empatía y todo miramiento por el otro y la otra, y; en el ámbito comunitario- político la sororidad.

\section{Un territorio puede ser pensado como un paisaje}

Un territorio puede ser pensado como un paisaje, puede ser la tierra en la que se emplaza, la gente que lo habita, la idiosincrasia, las relaciones de poder, la combinación de cada uno de nuestros puntos de vista.

Imaginen una vía que separa un barrio.
Alrededor casas de chapa y más atrás, se alzan unos robustos blocks de vivienda sociales. Imaginen transcurrir sus días cruzando esa vía, donde se acumulan restos de cereales y desechos de casas linderas. En ocasiones, algún grupo de caras conocidas te saludan, te hacen una broma. En otras, preferís no cruzar por ahí. No hay cloacas, ni gas natural. No llegan las líneas de teléfono fijo, ni cable y el colectivo más cercano pasa a cuatro cuadras, pero por las noches desvía su recorrido, alejándose del barrio.

Este paisaje fue el territorio que durante cinco años alojó nuestra práctica. Era las vecinas que nos saludaban todas las mañanas y los días de lluvia nos llevaban torta frita. Los pibes y las pibas que nos gritaban "seño", "profe" en la calle y preguntaban "cuándo hacemos algo". Chistes, risas, desencuentros. Pero también era ese pasillo por el que no podíamos pasar, ese rancho que teníamos que esquivar, ese pibe que no nos saludaba porque estaba en un maneje. $\mathrm{Y}$ además era todo eso que pasaba cuando caía la noche o el fin de semana y los actores "externos", las instituciones públicas, etc., no estábamos mirando. Una especie de "mundo íntimo" del barrio, que no paraba de estallar públicamente.

Los sujetos y sus territorios son co-producidos por cada época y por el discurso de cada forma de gobierno (Segato, 2013). Las primeras villas de emergencia nacen en los '50, como resultado del proceso de urbanización de las políticas económicas de sustitución de importaciones y desarrollo industrial del primer peronismo. Estaban pobladas por migrantes internos, y eran sitios pensados como lugares transitorios, de emergencia, "la villa funcionaba, al menos 
imaginariamente, como la escala hacia un lugar mejor" (Natanson, 2010).

A principios de los '80, surgen los asentamientos: la ocupación organizada de tierras, que tras el fin de la dictadura se multiplicó rápidamente. Los asentamientos son villas que se asumen ya no como de paso, sino como permanentes. El ideal de la ciudad homogénea y el ascenso social que funcionó desde fines del Siglo XIX entra en crisis y se transforma de la mano de las políticas neoliberales que generan desigualdad, el aumento del crimen y la proliferación de las urbanizaciones cerradas, tributario a la lógica privatista de los años '90 donde lo público- estatal se representa negativamente.

El resultado de estos procesos es la cristalización de formas de segregación socio espacial que se naturalizan. La noción de inseguridad, fogoneada por las clases dominantes y sus medios de comunicación, sirvió como excusa para justificar el incremento de la urbanización privada cerrada, tal como lo afirmaba Dammert (2001) y el abandono del espacio público por estar identificado como territorio de la criminalidad, con la consecuente pérdida de lazos comunitarios entre vecinos.

En la actualidad, la territorialidad se entiende como relación dinámica entre los componentes sociales (economía, cultura, instituciones, poderes) y aquello que de material e inmaterial le es propio al territorio donde se habita, se vive, se produce (Dematteis y Governa, 2005). Pero los territorios son también (en virtud de las transformaciones globales de las últimas décadas) carriles extensibles de identidad común e intereses compartidos (Segato, 2013).
El territorio, dice Segato (2008), es espacio marcado con los emblemas identificadores de su ocupación por un grupo específico, e inscribe con sus características la identidad de ese grupo que lo considera propio y lo transita libremente. Afirma, asimismo, que en el mundo de hoy sería posible decir que hay un nuevo proceso en curso en lo que respecta a la territorialidad entendida como experiencia particular, histórica y culturalmente definida. El territorio no se encuentra ya constituido por un espacio físico, sino por la red extensible de los cuerpos, de manera que los rasgos identitarios y las estrategias de marcación de los cuerpos, que garantizan pertenencia y filiación, se configuran como centrales.

Esta nueva territorialidad es la condición para lo que esta autora llama las nuevas formas de la guerra: el cuerpo inscripto como territorio y su ambiente espacial inmediato se constituyen en el campo de batalla de poderes en conflicto, pero también en "el bastidor donde se cuelgan y exhiben las señas de su anexión" (Segato, 2013). No debería extrañarnos, entonces, el uso de términos tales como bunker o soldadito propios de la jerga bélica.

La exaltación de los rasgos imaginarios que constituyen la identidad y las marcas del territorio, se observa muchas veces en el relato que el grupo de niñas con el que trabajamos hace de las disputas cotidianas del barrio:

"Seño jno la vas a invitar a M! jvive en el F (zona del barrio), son re choros ahí!" (M, 14 años).

"Las de calle E... se tiñeron todas de rojo el pelo, parecen unas trolas" 
(L, 12 años).

"Los de atrás de la vía son re atrevidos, no hay que darles cabida, por eso lo agarró a los tiros, cada vez que cruzan para acá se la dan” (S, 11 años).

En el marco del taller con las niñas, la complejidad del territorio se filtra constantemente en las conversaciones y pasa sin alarmas. Es marco y referencia. Términos como soldadito, transa, aparecen mezclados con vestidos de quince y la discusión por el chico más lindo de la escuela. Su propia identidad está jugada en eso. El territorio, sus emblemas, son cargados en sus cuerpos. En las redes sociales y en sus producciones dentro del taller, se identifican con sus nombres seguidos de las iniciales del barrio, por ejemplo, Yamila del BLP (Yamila del Barrio La Plata $\left.{ }^{1}\right)$. El barrio sella su identidad, la marca.

Las personas son depositarias y portadoras del territorio y eso se profundiza cuando hablamos de las mujeres, ya que existe una continuidad cognitiva entre cuerpo de mujer y territorio. Los cuerpos de las mujeres históricamente han constituido lugares privilegiados para el despliegue de las técnicas y relaciones de poder. Las condiciones sociales e históricas del capitalismo precisaron a estos cuerpos como terreno de explotación a fin de su conquista, como medios para la reproducción y acumulación del trabajo.

En las nuevas formas de la guerra, dice Segato (2013), la violencia contra las mujeres ha dejado de ser un efecto colateral y se ha transformado en un objetivo estratégi-

\footnotetext{
${ }^{1} \mathrm{El}$ nombre de la niña y del barrio con ficticios, usados solo a modo de ejemplificación.
}

co de este nuevo escenario bélico. Las chicas cargan en sus cuerpos información sensible (Colectivo Juguetes Perdidos, 2016), no solo de esas violencias, sino también de las que suceden puertas adentro. Tironeadas por mandatos sociales, sueñan con ser bailarinas o peluqueras. "Qué hermosa es esa mina”, dice M mientras mira un video de reggaetón donde la bailarina principal mueve la cola y está peinada con unas trenzas. S le cuenta que ella sabe hacer esas trenzas -le enseñó la prima- e improvisan una clase de peluquería, mientras se imaginan cómo será la vida de esa joven que "no vive en la villa" y que no se tiene que preocupar más que por "ser linda".

La mayoría de las niñas cuidan de sus hermanitos, y desde muy pequeñas se les trasmite que las mujeres son las responsables de la casa y la cría. "No puedo ir al taller seño, mi mamá se fue al médico con X y me tengo que quedar a cuidar al bebé", me dice L. Al preguntarle por qué no lo cuida su hermano mayor, responde exaltada: "Sos loca, seño, M es varón, cómo va a cuidar al bebé".

Sus cuerpos son mirados, criticados, estigmatizados, deseados, golpeados. Son ellos mismos territorios de las disputas y lienzo donde los "hijos del patriarcado" demuestran su masculinidad. Describen escenas donde se pasean por delante de grupos de chicos esperando que con sus palabras (verdadero ejercicio de la violencia machista, disfrazados de cumplidos) sancionen si son lindas, si están bien vestidas, si son mujeres. Sorprendentemente, perra es una de las palabras más esperadas.

La precariedad de los lazos sociales, impronta del neoliberalismo y también del patriarcado, se manifiesta entre ellas como 
traición. No podés contar con nadie que te dé una mano. En múltiples ocasiones hablan de la noción de amistad como algo frágil, la otra puede transformarse inmediatamente en enemiga. Los conflictos se resuelven a los golpes, no hay posibilidades de acuerdo, aquellas que hoy son amigas, mañana pueden golpearse sin miramientos por una remera copiada o por una mirada que es leída como malintencionada.

¿Con qué coordenadas podemos pensar estas violencias entre ellas? ¿Es acaso crueldad?

\section{Sobre la crueldad}

Los barrios hoy son más complejos, dice el libro ¿Quién lleva la gorra? del Colectivo Juguetes Perdidos (2016). Que sean más complejos significa que en ellos existe disponible más información sensible, más variables que explican al mundo, más elementos que pueden provocar la violencia. Cualquier pequeño desborde manifiesta la precariedad de las instituciones (que tienen como función regular la vida) y también la de los lazos y las redes cotidianas. En todo esto hay un trasfondo sensible de miedo, en cualquier momento el o la de al lado se puede convertir en una amenaza, el individualismo y el "sálvese quien pueda", valores fundamentales del liberalismo, han calado hondo en las subjetividades. Pedagogía de la crueldad, dice Segato.

Si hablamos de crueldad más allá de la pulsión, como dispositivo, Fernando Ulloa (2009) afirma que la crueldad es un dispositivo socio cultural, diferenciando de esta manera agresión/agresividad de crueldad: hay una diferencia sustancial entre lo que es la agresión como heredada del hombre, de toda la evolución de las especies, de la lucha por la vida, que es una expresión del instinto. El instinto no es cruel, en todo caso está sujeto a la ley de la sobrevivencia, no es cruel. Puede ser feroz pero no es cruel.

El desarrollo de la crueldad para Ulloa, tiene como antecedente en la constitución inicial del sujeto, la falencia de la ternura como primer anidamiento, como primer amparo que recibe el recién nacido o la recién nacida, pero este origen no signa el futuro despliegue de la crueldad, para su desarrollo, serán necesarios dispositivos socioculturales posteriores que, o bien no reparen ese origen fallido de la subjetividad, o lo acrecienten. El cachorro verá incrementada su instintividad astuta y agresiva en caso de encontrarse en el desamparo.

Rita Segato (2014) habla, en este sentido, de una pedagogía de la crueldad en nuestra sociedad, donde se enseña a no tener miramientos por el otro,

Por qué la pedagogía de la crueldad, por qué la gratuidad de la crueldad. Me parece que estamos en una fase del capitalismo al que le interesa tener sujetos no sensibles, sin empatía. $\mathrm{Y}$ esta etapa, donde el enriquecimiento y la acumulación se dan por despojo, donde el mercado es global; en esta abolición de lo local, que es la abolición de las relaciones interpersonales, de la propia empatía; es necesario entrenar a los sujetos para 
esa distancia, para esa crueldad, para la no identificación de la posición del otro y la no relacionalidad. Esa pedagogía de la crueldad es funcional a esta fase del capital.

Atravesando el salón, M elige su canción favorita en el equipo de música, es un reggaeton: "Baila todas las canciones y tiene un swing cuando se mueve, y no tiene amigas, deja que todas la envidian...", suena la canción. $\mathrm{M}$ realmente no tiene amigas, me dice que no se puede confiar en nadie. Los lazos de amistad entre ellas parecieran ser, en general, muy débiles y cambiantes, casi utilitarios. "Quien hoy es tu amiga, mañana es tu enemiga", dice con desconfianza. Suelen resolver los conflictos entre ellas con insultos e incluso llegan a golpes y rasguños. El motivo de las peleas muchas veces es un muchacho, otras el "me miró mal" y en ocasiones la ropa.

La crueldad se encuentra favorecida por la extensión de "lo cruel" (Ulloa, 2009) en la sociedad y de la violencia cotidiana en el territorio, todos elementos que tienden al borramiento del otro como semejante, ubicándolo como objeto o rival. Pero estas niñas no son crueles, en todo caso podríamos ubicar algunos actos crueles, así como también otros que responden a la más clara ternura.

Lo que sucede es que, en este contexto cruel, donde no cuentan con demasiados mecanismos comunitarios para elaborar su agresividad constitutiva y donde las formas de lo social propician la posición imaginaria de rivalidad con las otras, las agresiones se intensifican y se presentan como crueles.

La agresividad es en sí necesaria en el desarrollo subjetivo. Para autores como Winicott (1950- 1955), cierta agresividad posibilita el proceso de diferenciación, y para Lacan (1948) es fundamento de la constitución del yo. Aparece en el estadio del espejo frente a la fragmentación corporal, como la respuesta de separación del sujeto de la imagen que lo aliena. Cuando Lacan (2014) desarrolla el estadio del espejo dice que el niño niña en su inmadurez neurológica, cuerpo despedazado, se observa en el espejo y, con júbilo, se ve uno, unido, pero no sin antes ver al adulto- adulta que le sostiene frente al espejo, es decir la constitución del yo va a estar apuntalada en el otro que me sostiene. La idea de Yo es otro- o en el caso de las niñas, otra- resulta de la identificación con la imagen alienante, por la que se estructura el narcisismo y se origina el yo. Esa alienación del sujeto, dice Masotta (2008), a la imagen del espejo es el fundamento de toda identificación.

Esta forma se cristalizará en la tensión conflictual interna al sujeto, que determina el despertar de su deseo por el objeto del deseo del otro-otra, apareciendo la competencia agresiva. En el campo especular se da la lucha de yo o el otro -otra, y no hay otra posibilidad. La agresividad es, entonces, correlativa de la estructura narcisista del sujeto constitutiva de la primera individuación, es decir, una "identificación primaria que estructura al sujeto como rivalizando consigo mismo" (Masotta 2008), tensión imaginaria que se reacomodará durante el complejo de Edipo y por la función del Ideal del yo. Qué sucede con el atravesamiento del complejo de Edipo de cada una de estas niñas, es un desarrollo que escapó a las posibilidades del dispositivo diseñado, pero lo que sí fue po- 
sible ubicar, son algunos elementos a nivel comunitario que favorecen la posición de rivalidad.

En su texto de 1948 sobre la agresividad, Lacan (2014) desarrolla una perspectiva sobre el papel de la agresividad en el malestar de la cultura. "Tesis V: semejante noción de la agresividad como de una de las coordenadas intencionales del yo humano, y especialmente relativa a la categoría del espacio, hace concebir su papel en la neurosis moderna y en el malestar de la civilización".

Lacan (2014) afirma que la agresividad en la sociedad de su época, y en la actual también, se encuentra justificada al confundírsela con la fortaleza, idea sostenida por los desarrollos darwinianos de la selección natural, los cuales no hacen otra cosa que "proyectar las predaciones de la sociedad victoriana y la euforia económica".

Miller (1991) dice,

En los tiempos anteriores, el hombre estaba más vinculado a la comunidad, a los demás, en los ritos ceremoniales, en la vida en común, la familia extendida restringía la agresividad. Y cómo la civilización moderna, en cambio, aísla al individuo y pone en evidencia su yo, esto refuerza su paranoia estructural (p. 14).

La posibilidad de elaboración comunitaria de la agresividad mediante los ritos y ceremonias se encuentra en nuestra actualidad altamente reducida por la fragilidad existente en los lazos. Se fortalece la posición imaginaria, la otra es mi enemiga y me amenaza: es ella o yo.

En este sentido, Rodriguez Costa (2016) se pregunta ¿Qué hace la sociedad moderna y occidental con la agresividad? Y se responde; invierte los términos: del desarrollo de la preocupación de dañar al otro-otra, en los términos de Winnicott, pasamos a la preocupación de que el otro-otra me dañe. Se constituye un dispositivo de transformación de la agresividad en violencia, en la medida en que todo lo que se reprime y no se elabora, no se elimina, sino que se actúa: la agresividad se proyecta al otro-otra y los actos de agresión se actúan sin que se los reconozca como tales.

Con respecto a las manifestaciones de agresión que los estereotipos tradicionales ofertan a las mujeres, Hidalgo Xirinachs (2002) dice,

Si se muestran contenidas y expresan la agresividad de forma indirecta, aparecen como "traicioneras" o incluso incapaces frente a las nuevas demandas que la realidad actual les exige. $\mathrm{Si}$, al contrario se muestran agresivas de forma abierta y directa, son descalificadas rápidamente como personas innecesariamente agresivas. Mientras que la agresividad asertiva en los hombres posibilita la autodeterminación o incluso la imposición violenta de los propios intereses y tiende a considerarse un comportamiento masculino legítimo, en las mujeres se mantiene una ambivalencia extrema al respecto.

Aunque en la actualidad se considere que la mujer puede ser agresiva en forma abierta y activa, en realidad, su agresión se vuelve ilegítima. Los componentes de la 
agresión que conforman aquellas actitudes que buscan la diferencia y la distancia frente al otro, se transforman de esta manera, para la sociedad y para cada mujer, en agresión violenta y autodestructiva.

Algunas escenas descriptas por las niñas podrían ser ubicadas como vinculadas a la posición de agresividad imaginaria donde se juega la ambivalencia: deseo a ese otro-otra y a la vez tengo que destruirle, porque me amenaza en mi supuesta unidad yoica.

D me cuenta: "...en clase me miraba mal, ya se venía haciendo la viva, así que la esperé a la salida y le arranqué todos los pelos, no se va a hacer más la viva conmigo" (D, 11 años). "Qué mirás” le pregunta desafiante J a L en el taller, "mirate el culo". "Yo me iba a hacer el aro también, pero lo tiene cualquier gil ahora" le dice D a L que acaba de estrenar piercing en la nariz. "Ahora me lo voy a hacer acá" (se señala el entrecejo).

En estos relatos, y en muchos otros similares, aparece lo especular como condición para la agresión. Un par de zapatillas nuevas, un jean, el pelo o las uñas se convierten en objetos de la otra que hay que destruir por no poder poseerlos. "Se compró las mismas zapatillas! La voy a recagar a trompadas" “¿Por qué?”, pregunto. “No entendés, seño, las mismas zapatillas!” (L, 10 años)

Estas escenas de agresión se intrincan con otras violencias, haciendo más compleja su lectura: $\mathrm{M}$ y $\mathrm{C}$ no se hablan en todo el taller, eran muy compinches hasta la semana pasada por lo que me llama la atención. Al terminar, cuando estamos solas, le pregunto a $\mathrm{M}$ qué pasó: me contesta que se enteró de que $C$ anda hablando mal de su hermano mayor. Ambas niñas pertenecen a familias antagonistas en el negocio de la venta del barrio y si bien conviven en el espacio del taller, los episodios de violencia entre los adultos o jóvenes de las familias no dejan de demarcar los límites y las posibilidades a la hora de vincularse.

Rita Segato (2013) plantea que la violencia ejercida sobre los cuerpos de las mujeres es un epifenómeno de la violencia entre bandas. Ambas violencias están vinculadas a la cultura machista y la agresión sobre estos cuerpos está enlazada con los ritos de masculinidad que desde las bandas se les exigen a los hombres. Hay que probar todo el tiempo que uno es "bien hombre" y en eso la dominación de la mujer y el uso de las armas son pruebas fundamentales. La misma autora habla del "mandato de masculinidad" (Segato, 2017): el varón debe demostrar su potencia a la banda y lo hace a través de la crueldad ejercida sobre los cuerpos feminizados (que no solo corresponden a los de las mujeres). La violencia hacia las mujeres se ejerce, además, con otras finalidades: disciplinar a la víctima, merecedora de castigo y como una forma de desmoralizar a los hombres que deberían estar tutelando esos cuerpos.

\section{Esos cuerpos}

Es gorda, fea y re puta dice L, respondiendo a la pregunta de por qué no le cae bien una chica del barrio. Así muchas veces se refieren las niñas a sus pares: gorda y puta son los epítetos más usados.

Fea - Gorda - Puta - tres adjetivos que se refieren al cuerpo de las mujeres, cuerpo-placer, cuerpo sexuado. El dominio sobre los cuerpos de las mujeres y las niñas, sobre su sexualidad, ha sido tarea funda- 
mental del sistema patriarcal capitalista desde sus inicios. Expropiación, dominio, control, el sistema define que pueden hacer con sus cuerpos, cómo, cuándo y con quién. El cuerpo como territorio de conquista, sobre el cual se manifiestan las diferentes violencias que los rodean.

"Anda con todos, seño, se la pasan. (...) Nadie la quiere”. (M, 12 años) "Se pone esos shorts de trola y después no quiere que la violen, en el baile la toquetean todos". (L, 13 años).

Esto sostienen las niñas del barrio. ¿Cómo es posible que una niña, que experimenta sobre su propio cuerpo la opresión de esta ideología reproduzca esos enunciados? ¿Qué lleva a las mujeres a competir, rivalizar, culpabilizarse, tomando como propias las palabras del patriarcado?

Franz Fanón (2011) en su libro Piel negra, máscaras blancas, narra la condición de las personas de raza negra que viven en las colonias francesas. Habla de la opresión que el sistema colonial les impone, la represión y menosprecio de su cultura propia y la violenta instauración de la cultura francesa en su lugar. Frente a tremenda violencia, uno sospecharía un gran rechazo de la colonia por parte de los colonizados, pero lejos de esto, el autor narra un hecho que lo sorprende: la identificación de los negros y las negras con sus colonizadores, identificación que resulta en un desprecio por sus propios pares y un abrazar la cultura y las formas francesas. Habla de una identidad colonizada. ¿Qué mecanismo tiene que operar para que un sujeto asuma íntimamente el poder que lo oprime, aun en contra de su propia supervivencia?

Desde un primer acercamiento podríamos pensar que el poder es un fenómeno externo al sujeto, pero Butler (1997), siguiendo a Foucault, Althusser y Hegel, dirá que el poder, al mismo tiempo que subordina al sujeto, lo constituye desde dentro, entregando la condición de su existencia y dibujando la trayectoria de su deseo. Para el sujeto, el poder tendrá entonces dos dimensiones: por un lado, implica sumisión, ejerce presión desde afuera, lo subordina. Por otro lado, se plantea necesario para la constitución subjetiva, es la condición de su fundación.

Cuando las categorías sociales, como la de género, garantizan una existencia social reconocible y perdurable, la aceptación de estas categorías, aún si operan al servicio del sometimiento, suelen ser preferibles a la ausencia total de existencia social. Es así como se explica que las mismas mujeres oprimidas por el discurso patriarcal asuman como propias estas categorías y las reproduzcan. Esta reproducción, asimismo, se ve garantizada por el rol de género de cuidadoras de los hijos y las hijas. Capitalismo y patriarcado funcionan como una máquina perfectamente engarzada que sostiene la reproducción del sistema desde el inicio de la vida.

Butler (1997) agrega una pregunta a sus desarrollos, ¿cómo adoptar una posición de oposición ante el poder aun cuando se deba reconocer que toda oposición está comprometida con el mismo poder al cual nos oponemos? Aquí la pregunta nos sumerge en el mundo político, donde encontramos un mecanismo restitutivo que, a partir del 
reconocimiento de la opresión, nos coloca como pares y nos permite la transformación de nuestras condiciones de existencia. Asimismo, al construir otras categorías que garantizan la existencia social del sujeto, ofrece alternativas al modo de lazo del patriarcado-capitalismo: la sororidad.

\section{La casa del amo no se desarma con las herramientas del $\mathrm{amo}^{2}$}

Este grupo de niñas transcurre sus días en un territorio fuertemente signado por el mandato de masculinidad, donde, por ejemplo, aquella vecina que cayó víctima de una red de trata es responsable por "haberse ido con los tipos" o porque "siempre fue medio puta”, según sus palabras. Una y otra vez aparece la culpabilización a la víctima y la dificultad para empatizar y pensar a la otra como una semejante.

¿Es posible para estas niñas vincularse de otra manera, una manera que tienda a la construcción colectiva, fundada en la empatía y solidaridad y no ya en el destrato y la competencia entre ellas? Las luchas feministas nos han proporcionado un concepto en este sentido: sororidad. Pero a su vez estos dispositivos sociales deben poder sostenerse en un mecanismo que garantiza la capacidad del desarrollo de la empatía en el cachorro humano: la ternura.

Para Fernando Ulloa (2009) la ternura es la coartación, el acotamiento, la limitación, del fin último de la pulsión. Con esto se refiere a que una madre ${ }^{3}$ no sobreagrede

\footnotetext{
2 Tomamos el título de los comentarios presentados por Audre Lorde en el "Encuentro personal y político" del Congreso del Segundo Sexo, Nueva York, 29 de septiembre de 1979.

${ }^{3}$ Se plantea el termino madre como una función, no
}

ni sobreexita a su niño o niña. La ternura es una coartación de la pulsión sexual y de la pulsión agresiva, o de la agresión y de la excitación dentro de la pulsión sexual.

Esa coartación significa, también, cierta coartación de sublimación, de la cual nacen dos cosas: la empatía y el miramiento. En cuanto a la empatía, la madre debe poder leer en ese llanto qué es lo que el cachorro necesita, aun cuando este tiene apenas horas. La madre sabe por qué el niño o la niña llora, y ese saber garantiza el suministro. Con respecto al miramiento, Ulloa (2009) plantea que es "mirar con interés", con consideración seria, mirar con interés amoroso, a alguien que es reconocido como un sujeto distinto, sujeto otro, sujeto ajeno. "Entonces, si la empatía garantiza los suministros necesarios a la vida, el miramiento garantiza el gradual desprendimiento de este sujeto como sujeto autónomo" (Ulloa, 2009).

La ternura es una situación de tres lugares. Esa coartación del fin último se hace en función de la presencia social, que no es necesariamente el padre, ya que la madre tiene internalizada, como sujeto social que es, a ese tercero. Se evidencia la diferencia con la situación de la encerrona trágica, donde no hay tal tercero de apelación, sino una situación social dual de amo-esclavo, de victimario a víctima.

La ternura tiene tres suministros básicos: el abrigo, frente a los rigores de intemperie; el alimento, frente a los rigores del hambre; y el buen trato. El buen trato es trato con arte, es trato pertinente, pero fundamentalmente es algo que tiene que ver con la donación simbólica, dice Ulloa (2009). La

necesariamente ocupada por la progenitora u otra mujer. 
ternura existente será, entonces, el basamento sobre el cual se podrá construir cualquier otro dispositivo cultural que tienda a la instalación de la otra como semejante. La renuncia pulsional que ésta implica, es la posibilidad de establecer lazos solidarios entre las mujeres.

La sororidad, dice Lagarde (2014), es una dimensión ética, política y práctica del feminismo contemporáneo. La sororidad es la conciencia crítica sobre la misoginia (el otro lado del machismo), sus fundamentos, prejuicios y estigmas. Es el esfuerzo personal y colectivo de desmontar, en la subjetividad, la cultura patriarcal, y de generar una transformación solidaria de las relaciones con las mujeres, las prácticas sociales y las normas jurídico políticas.

Al enfrentamiento misógino entre mujeres, la autora lo denomina enemistad patriarcal. En esta idea es posible pesquisar cómo los dispositivos del patriarcado se montan sobre la agresividad especular propia del desarrollo del yo, en una estructura social que no solo no propone mecanismos de elaboración de la misma, sino que la profundiza y hace usufructo de ella. Los objetivos de este dispositivo de enemistad son: la competencia rival, y la desidentificación de género entre las mujeres como mecanismos de reproducción patriarcal.

"A S también le gusta J., pero él me dijo que a esa gorda no la toca, yo soy más linda", dice $\mathrm{M}$, refiriéndose a un muchacho del barrio que le gusta. Este tipo de comentarios donde se descalifica a la otra y se siente orgullo por haber sido la elegida por la fratría es muy común y está totalmente naturalizado entre las chicas, al marcar algo de esto la respuesta es: “¡Ay! ¡bueno, seño! Él lo dijo", seguido de una sonrisa que se disimula en un intento de ocultar la satisfacción presente en esas escenas.

La sororidad se construye mediante la adscripción a ese pacto que parte de la toma de conciencia de las necesidades comunes y, a su vez, particulares de las mujeres, así como del empoderamiento y la solidaridad surgida entre ellas. Una solidaridad que, a su vez, nace de la conciencia femenina de su opresión dentro del orden patriarcal. Cuando viene a buscar a la niña al taller y en su presencia, la madre de E me cuenta que a partir de darse cuenta de que a todas las mujeres del barrio le pasa lo mismo que a ella (golpes por parte de su marido), ya no se mete adentro ni sube la radio cuando algún vecino está golpeando a su mujer. En ocasiones, ahora llama a la policía y está atenta a poder ayudar. Antes, en cambio, creía que los golpes les pasaba porque hacían enojar a sus maridos. Encuentro en esta escena un pequeño esbozo de lo que puede ser el ejercicio de la sororidad.

De esta manera es posible ubicar la necesidad de la construcción de dispositivos comunitarios que, a partir de recuperar las propias experiencias locales de cuidado y organización, instale la práctica de la sororidad como una toma de conciencia sobre la opresión y pueda horadar, aunque sea mínimamente, la gigantesca construcción de la pedagogía de la crueldad.

Queda quizás como pregunta, cuál es el horizonte subjetivo de construcción de sororidad en estas niñas y su vínculo con la ternura como primer anidamiento, ya que para que algo de la sororidad se ubique en un lugar de posibilidad, debe existir primero un Otro consistente que aloje, para lue- 
go matar al padre del discurso patriarcal y no ocupar su lugar.

\section{Reflexiones finales}

Hemos emprendido este escrito con el objetivo de visibilizar las prácticas de niñez de un grupo de niñas de un barrio, entendiendo que como sujeto colectivo la niña ha sido invisibilizada para las políticas de estado, las comunidades y la academia.

En el encuentro con este grupo llamaron la atención los niveles de agresión con los que se relacionaban entre ellas. Fue, quizás, nuestro propio prejuicio sobre cómo deberían ser las niñas, lo que nos llevó a poner la mirada en esto. El patriarcado como dispositivo funciona dentro de cada uno y cada una de nosotras. Pero fue también el proceso de reflexión el que permitió desmontar prejuicios e intentar comprender las lógicas propias de este grupo.

En el transcurso del taller nos preguntamos si la noción de agresividad como constitutiva y necesaria a la hora del desarrollo del yo y la autonomía, era suficiente para explicar este modo de lazo. Nos encontramos, entonces, con que ciertas características, como la labilidad de los lazos, el cuerpo sexuado como objeto de las agresiones y el rasgo de crueldad de alguna de ellas, desbordaban la posibilidad de comprensión desde este registro únicamente. Encontramos la clave en el discurso de las niñas. En sus enunciados aparecían constantemente referencias a la violencia del territorio.

Los planteos de Rita Segato nos permitieron comprender cómo se articula territorio - violencia - género y la exacerbación de los rasgos imaginarios. En la forma actual de la territorialidad, donde los territorios son carriles extensivos de identidades compartidas, los rasgos identificatorios se llevan en el cuerpo y la agresión no se dirige solo a ese cuerpo, sino a todos y todas aquellas que conforman el agrupamiento que sostiene esa identidad. En esta nueva forma de la guerra, el cuerpo de las mujeres es territorio privilegiado, donde en virtud del mandato de masculinidad, los hombres pretenden a demostrar su valor a la fratria. La razón por la que esto aparece asumido por las niñas está vinculada a la emergencia misma del sujeto, constituido a partir del discurso del otro, lo que le da la posibilidad de existencia social, otro que en este caso se trata del patriarcado.

En cuanto al manejo social de la agresividad, las formas de capitalismo actuales no promueven el desarrollo de dispositivos comunitarios de elaboración, por lo que la misma se reprime y se actúa, transformándose en violencia. Específicamente para las mujeres, las formas de socialización contemporáneas cuentan con una doble versión: por un lado, propician el desconocimiento de la agresividad -según el estereotipo de la madre cuidadora-, a la vez que, en virtud de la aceptación de la inferioridad de lo femenino como premisa y la búsqueda de las mujeres de ser elegidas por la comunidad de hombres, se favorece la rivalidad y la competencia. De esta manera, se refuerza la agresividad imaginaria correlativa a la estructura narcisista del sujeto.

Asimismo, no podemos decir que estas niñas sean crueles. Asumen en ocasiones posiciones crueles, favorecidas por los dispositivos socioculturales, que no solo no reparan allí donde algo de la ternura 
falló, sino que tienden a borrar la noción de semejante, favorecer la rivalidad y desarticular los lazos locales. Es por eso que consideramos necesaria la construcción de dispositivos comunitarios, al modo de suplencias, que recuperen las potencias de cuidado territorial, fundadas en la ternura en sus tres elementos: abrigo, alimento y buen trato; y con la sororidad como horizonte y modelo político de destitución del discurso patriarcal.

En los últimos años, se han dado una serie de transformaciones en nuestra sociedad que tienen como corolario la apertura a nuevos modos de crianza y vínculos, críticos de los modelos tradicionales del patriarcado, donde los niños y las niñas son ubicados como sujetos de derecho de un modo igualitario, y se deconstruyen los roles típicos asignados al género.

Estas modificaciones se dan en un tiempo notablemente acotado y de la mano de movimientos como el Ni una menos, o el debate sobre la Ley de aborto legal, seguro y gratuito, que tienen sus orígenes en la clase media de la sociedad. Es posible que estos procesos modifiquen los modos de constitución subjetiva de este grupo de niñas, lo que solo podrá producirse lentamente.

En este sentido, se hace imprescindible no dejar de observar la categoría de clase y el modo en que se entrama con la de género. Se hace necesario para ello, conocer las diferentes realidades de las mujeres que no cuentan con los privilegios de pertenecer a cierta clase o incluso franja etaria, como en este caso, donde se agrega la condición infantil (Talpade Mohantyn, 2008). Pero lo más importante es comprender que la complejidad no está dada por la sumatoria, es decir opresión de genero + clase, + edad, sino que constituye realidades completamente diversas. A este proceso se lo denomina descolonizar al feminismo.

\section{Referencia}

- Burin, M. (1996) Género y Psicoanálisis: Subjetividades femeninas vulnerables, en Burin \& Bleichmar (comp.) Género, psicoanálisis y subjetividad, Buenos Aires: Paidós.

- Butler, J. (1997) Mecanismos psíquicos del poder. Teorías sobre la sujeción. Madrid: Cátedra.

- Colectivo Juguetes Perdidos (2016) ¿Quien lleva la gorra? Violencia, nuevos barrios, pibes silvestres. Buenos Aires: Tinta Limón.

- Dammert, L. (2001). Construyendo ciudades inseguras: temor y violencia en Argentina. Santiago: EURE.

- Dematteis, G. y Governa, F. (2005) Territorio y territorialidad en el desarrollo local. La contribución del modelo slot. En Boletín de la A.G.E. N. ${ }^{\circ}$ 39, págs. $31-$ 58. Turin: Departamento Interateneo Territorio. Politécnico y Universidad de Turín.

- Fanon, F. (2011) Piel negra, máscara blanca. La Habana: Caminos.

- Fernández, A. M. (1994) La invención de la niña. Buenos Aires: UNICEF.

- Giberti, E. (2003) La niña: para una gnoseología de la discriminación inicial, recuperado de www.evagiberti.com. Fecha de recuperación: 22 de Agosto de 2015.

- Hidalgo Xirinachs, R. (2002) Sexualidad, agresión y autonomía en la mujer: Contribuciones psicoanalíticas actuales. 
En Actual. psicol. [online]. , vol.18, n.105. Mexico.

- Lacan, J. (2014) La agresividad en Psicoanálisis. En Escritos 1. Buenos Aires: Siglo XXI.

- Lagarde, M. (2004) La multidimensionalidad de la categoría género y del feminismo en Metodología para los estudios de género. Instituto de Investigaciones Económicas, Universidad Nacional Autónoma de México. México.

- Lagarde y de los Ríos, M. (2014), El feminismo en mi vida: hitos, claves y topias. Mexico: Horas y horas.

- Masotta, O. (1992). Lecturas de psicoanálisis. Freud, Lacan. Buenos Aires: Paidós.

- Miller, J. A., (1991) Agresividad y pulsión de muerte. Medellín: Fundación Freudiana de Medellín.

- Natanson, J. (2010) Villas y asentamientos. En Diario Página/12. Edición del 26 de diciembre. Recuperado de https://www.pagina12.com.ar/diario/ elpais/1-159346-2010-12-26.html. Fecha de recuperación: 20/02/2017

- Rodríguez Costa, L. (2016) Violencia: la disputa social por los destinos de la agresividad. Trabajo presentado en $2^{\circ}$ Encuentro Nacional por la Niñez. Panel sobre Violencias, 11 y 12 de Noviembre, Rosario. Inédito.

- Segato, R. (2008) La faccionalización de la república y el paisaje religioso como índice de una nueva territorialidad. En Alonso, A. (Comp.) América Latina y el Caribe. Territorios religiosos y desafios para el diálogo, Buenos Aires: CLACSO, Consejo Latinoamericano de Ciencias Sociales.

- Segato, L. R (2013) La escritura en el cuerpo de las mujeres asesinadas en Ciudad Juárez. Buenos Aires: Tinta Limón

- Segato, R. (2014) Entrevista: En los medios existe una pedagogía de la crueldad. En Noticias de la Facultad de Periodismo y Comunicación Social de la Universidad Nacional de La Plata, 06 de Octubre de 2014. Recuperado de http:// perio.unlp.edu.ar/node/4602 . Fecha de recuperación: 20 de Diciembre de 2016.

- Segato, R. (2017) Conferencia en Mesa Debate Género y violencia, 17 de Agosto, Rosario: sin editar.

- Ulloa, F. (2009) La crueldad-parte I. Clase del sábado 11 de Diciembre de 1999. Recuperado de http://antares-porvenir.blogspot.com.ar/2009/08/la-crueldad-fernando-ulloa.html. Fecha de recuperación: 02 de mayo de 2017.

- Talpade Mohantyn, C. (2008) Bajo los ojos de Occidente: Academia feminista y discurso colonia. En Suárez, L.y Hernández, R. A. (eds.) en Descolonizando el feminismo. Teorías y prácticas desde los márgenes. Madrid: Cátedra. 